\title{
Procarbazine and antidepressants: a retrospective review of the risk of serotonin toxicity
}

\author{
Shawna L. Kraft ${ }^{1,2}$, Nicole M. Baker ${ }^{3}$ Julia Carpenter ${ }^{4}$ and Jolene R. Bostwick ${ }^{1,2 *}$ \\ 'Department of Clinical, Social, and Administrative Sciences, College of Pharmacy, University of Michigan, Ann Arbor, MI, USA \\ ${ }^{2}$ Department of Pharmacy Services, University of Michigan Health System, Ann Arbor, MI, USA \\ ${ }^{3}$ Meijer Pharmacy, Mason, MI, USA \\ ${ }^{4}$ College of Pharmacy, University of Michigan, Ann Arbor, MI, USA \\ *Correspondence to: \\ Abstract
} Department of Pharmacy Services, University of Michigan Health System, 1500 E. Medical Center Drive, 9D9814 University Hospital, Ann Arbor, Ml 481090018, USA. E-mail: jkingsbu@ med.umich.edu

Received: 18 February 2013

Revised: 15 July 2013

Accepted: 23 July 2013

\begin{abstract}
Background: Procarbazine is an anticancer agent that also inhibits monoamine oxidase, an enzyme responsible for the metabolism of various catecholamines, including serotonin.

Methods: A retrospective chart review of lymphoma patients who were treated with both procarbazine and an antidepressant, as well as procarbazine alone, was performed to determine if signs and symptoms of serotonin toxicity were present.

Results: A total of 65 patients received procarbazine between 2004 and 2010 and were eligible to be included in the study. Twenty-six of these patients received an antidepressant in combination with procarbazine, with selective serotonin reuptake inhibitors being the most common type of antidepressant. No patients in the study were diagnosed with serotonin toxicity, nor did any meet Hunter's diagnostic criteria for serotonin toxicity. Diarrhea, tremor, and shivering were the symptoms from Sternbach's criteria that were further analyzed, with diarrhea occurring $8.54 \%$ of the time, tremor occurring $5.53 \%$ of the time, and shivering occurring $2.51 \%$ of the time in patients who received an antidepressant with their procarbazine. Despite these symptoms, the diagnosis of serotonin toxicity according to Sternbach's criteria was determined to be unlikely.

Conclusions: In this small sample of patients treated with procarbazine plus an antidepressant (most typically SSRIs), there were no reports of serotonin toxicity, nor did any patients demonstrate symptoms consistent with serotonin toxicity. The authors urge clinicians to ensure depression is adequately managed in cancer patients who are undergoing procarbazine therapy, starting with typical first-line antidepressant agents.
\end{abstract}

Copyright () 2013 John Wiley \& Sons, Ltd.

\section{Introduction}

In addition to its anticancer mechanism, procarbazine, a drug used in the treatment of Hodgkin's and non-Hodgkin's lymphoma and various brain tumors, has a metabolite demonstrating weak serotonergic activity through nonselective inhibition of the monoamine oxidase enzyme $[1,2]$. The combination of a monoamine oxidase inhibitor (MAOI) with a selective serotonin reuptake inhibitor (SSRI) is the most common cause of serotonin toxicity (ST), a toxidrome that can be life-threatening [3]. The question remains as to the clinical significance of this interaction among patients treated with procarbazine. Literature searches for reports of patients who developed ST from concomitant treatment with procarbazine and an antidepressant revealed no cases of an interaction. Further, interaction-checking databases provide inconsistent results when the combination of an antidepressant with procarbazine is queried, ranging from no interaction to contraindicated [3-6]. When a risk of using concomitant therapy is recognized, this risk is cited as ranging from theoretical to established [3-6]. Based upon this information, it appears that the concern over an interaction is based solely on procarbazine's classification as a MAOI with no supporting clinical accounts, and the true clinical significance of this interaction is unclear. The information provided by the manufacturer in regard to this interaction did not contain any studies with procarbazine and antidepressants [Sigma-Tau Pharmaceuticals, e-mail communication July 2013]. The substantial difference in these results provides practitioners with conflicting information and no evidence-based guidance for treating depression in patients receiving treatment with procarbazine. This is particularly problematic given the prevalence of comorbid mood disorders in cancer patients. More specifically, a 2009 review article published by Roper, et al. evaluated 35 studies of patients treated for Hodgkin's disease and estimated the prevalence of anxiety and/or depression among this population to be up to $50 \%$ within the first year after diagnosis [7]. This number is significantly higher than in the general US population, in which an estimated $18 \%$ suffer from 
anxiety and $9.5 \%$ from mood disorder each year [8]. Further, the majority of those experiencing mental illness are not treated or are inadequately treated [9], highlighting the urgency of addressing mood disorders and understanding the need for safe and effective treatments in cancer patients. Serotonergic drugs such as SSRIs and serotonin and norepinephrine reuptake inhibitors are the medications of choice for the treatment of depression [10-12]; however, clinicians may be hesitant to utilize these medications due to the theoretical risk of serotonin toxicity when these antidepressants are used in combination with procarbazine. Failure to treat depression in this population could have devastating effects, as cancer patients suffering from depression have an increased rate of relapse and are less adherent to antineoplastic therapy [10]. The objective of this study is to determine if concomitant treatment with procarbazine and an antidepressant places patients with cancer at risk for serotonin toxicity.

Serotonin toxicity generally occurs when serotonergic antidepressants are used in combination with other serotonergic drugs. ST is most likely to be fatal when SSRIs are co-administered with MAOIs [12]. Following the use of serotonergic agent(s), the onset of serotonin toxicity is rapid and typically occurs within the first 24 hours following initiation, change in dose, or overdose [13]. Data from the national poison data system from 2011 notes among 46,587 exposures to SSRIs, there were 1,623 mild outcomes, 134 major outcomes, and 11 deaths. The majority of fatalities reported were due to combinations of various agents [14]. The actual incidence of serotonin toxicity is not well characterized due to lack of recognition from variations in presentation, among other reasons $[15,16]$.

While the gold standard of diagnosing serotonin toxicity is via a toxicologist, the likelihood of serotonin toxicity may be more readily assessed using Sternbach's diagnostic criteria and the Hunter serotonin toxicity criteria (HSTC) [15,17], Sternbach's criteria is an older tool in which three or more of clinical features must be present to be considered positive for ST, as well as having a serotonergic drug recently added to therapy or an increase in dose. These clinical features include confusion, hypomania, restlessness, myoclonus, hyperreflexia, diaphoresis, shivering, tremor, diarrhea, or incoordination [15]. The primary shortcoming of this assessment is its lack of specificity because many of the clinical features overlap with symptoms seen in alcohol withdrawal states, catecholamine excess, antidepressant discontinuation syndrome, or anticholinergic delirium, for example [18]. Conversely, HSTC is a newer diagnostic technique that is considered a highly sensitive (84\%) and specific (97\%) approach to ST diagnosis when compared with the gold standard [17]. This method employs a series of decision rules to assess a patient for ST based on symptom presentation, which are summarized in Figure 1 [17] and is deemed to be the most accurate criteria available, using a more narrow range of clinical features, most namely, clonus $[16,18]$.

\section{Methods}

\section{Sample and procedures}

This retrospective cohort study was conducted at the University of Michigan Health System, which includes a 990 bed university hospital. This study protocol was approved by the University of Michigan institutional review board. Eligible patients included those with cancer at the University of Michigan Health System that were at least 18 years of age and had been prescribed at least once cycle of procarbazine alone for the control group, or those who were treated concomitantly with procarbazine and an antidepressant between 2004 and 2010. Patient demographics, procarbazine regimen, antidepressant regimen, comorbidities, and coadministration of additional serotonergic medications were collected. Medications that interact with CYP 2D6 or 3A4 enzymes should also be noted as they may impact the level of circulating antidepressant.

\section{Measures}

Each patient's multidisciplinary chart was reviewed for the signs and symptoms of ST. Because several conditions have features similar to ST, patients suspected to be positive for ST were further evaluated for symptoms that would indicate another condition. Because two sets of criteria are commonly used to diagnose ST, Sternbach's diagnostic criteria and the HSTC, as described earlier, both were used to evaluate patients for ST in this study.

\section{Analyses}

Categorical data were analyzed using $\chi^{2}$ or Fisher exact test. Continuous data were analyzed with the Student $t$-test

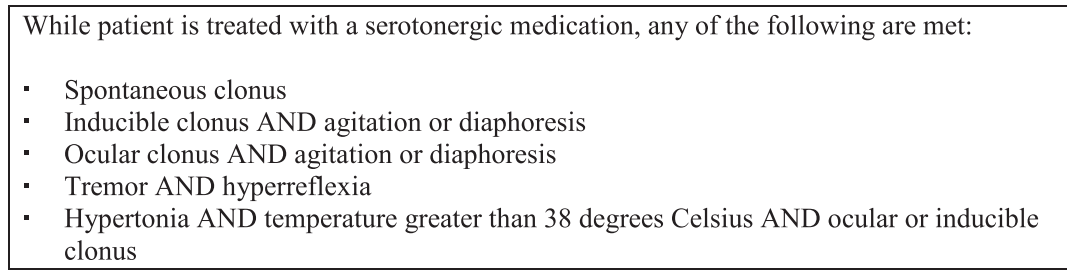

Figure I. Hunter serotonin toxicity criteria predictors of serotonin toxicity $[15,17]$ 
(for dichotomous variables) or Mann-Whitney $U$-test (for nonparametric variables). All tests were 2 -sided and $p$-values $<0.05$ were considered statistically significant. The model used for correlating serotonin toxicity symptoms was a generalized linear model with generalized estimating equations to take into account the fact that there could be multiple procarbazine cycles for a given patient, and thus, observations for the same patient could be correlated in each cycle. All analyses were conducted using SAS software, version 9.1 (SAS Institute).

\section{Results}

\section{Patient characteristics}

A total of 65 patients received procarbazine between 2004 and 2010 and were eligible to be included in the study. Twenty-six of these patients also received an antidepressant. There were slightly more males $(61.5 \%)$, patients with a stage 4 diagnosis $(48.7 \%)$, and more patients with lymphoma diagnosis $(53.8 \%)$ included in the study. The overall mean age was $57.7 \pm 16.7$ years. Patient characteristics based on cancer diagnosis are summarized in Table 1. Patients with lymphoma tended to be older, male, and have undergone more previous chemotherapy regimens than patients with brain tumors. The majority of lymphoma patients were stage 4 , whereas the patients with brain tumors were evenly distributed amongst stages 2, 3, and 4 . The average procarbazine dose in patients with a brain tumor was almost double the average daily dose of those patients with lymphoma. Patient characteristics comparing those that received an antidepressant to those that did not are summarized in Table 2. The patients who received antidepressant were more likely to

Table I. Comparison of patient characteristics based on diagnosis

\begin{tabular}{|c|c|c|c|}
\hline Variable & $\begin{array}{l}\text { Brain } \\
\text { tumor } \\
(N=30)\end{array}$ & $\begin{array}{l}\text { Lymphoma } \\
\qquad(N=35)\end{array}$ & P-value \\
\hline Age (mean) & $49.8 \pm 15.7$ & $64.4 \pm 14.7$ & 0.0003 \\
\hline Male (\%) & 53.3 & 68.6 & 0.3065 \\
\hline \multicolumn{4}{|l|}{ Stage at diagnosis (\%) } \\
\hline I & 0 & 2.9 & 1.000 \\
\hline 2 & 33.3 & 20 & 0.2658 \\
\hline 3 & 33.3 & 11.4 & 0.0393 \\
\hline 4 & 33.3 & 65.7 & 0.0130 \\
\hline \multicolumn{4}{|c|}{ Number of prior chemotherapy treatments (\%) } \\
\hline No prior treatment & 3.3 & 17.1 & 0.1124 \\
\hline I prior treatment & 36.7 & | 1.4 & 0.0203 \\
\hline 2 prior treatments & 46.6 & 22.9 & 0.0654 \\
\hline 3 prior treatments & 6.7 & 14.3 & 0.4370 \\
\hline$\geq 4$ prior treatments & 6.7 & 34.3 & 0.0135 \\
\hline $\begin{array}{l}\text { Number of procarbazine } \\
\text { cycles (median) }\end{array}$ & $2(1-3)$ & $2(1-4)$ & 0.6638 \\
\hline $\begin{array}{l}\text { Dose per day (mg) of } \\
\text { procarbazine (median) }\end{array}$ & $\begin{array}{l}66.1(55.8- \\
119.2)\end{array}$ & $\begin{array}{c}37.5(35.7- \\
50.0)\end{array}$ & 0.0001 \\
\hline Taking antidepressant & 33.3 & 45.7 & 0.4465 \\
\hline
\end{tabular}

have a diagnosis of lymphoma, have had two prior chemotherapy treatments, and be slightly younger compared with those who did not receive an antidepressant, although none of these differences were statistically significant. Of the 26 who received an antidepressant, SSRIs were the most common type (53.8\%), followed by either mirtazapine or trazodone (23.1\% and classified as other), followed by serotonin and norepinephrine reuptake inhibitors (19.2\%), and only one patient received a tricyclic antidepressant. No patients received MAOIs as treatment for depression. Specifically, seven patients received trazodone, six patients received citalopram, four patients received venlafaxine, three patients received sertraline, two patients received escitalopram, and one patient each received amitriptyline, mirtazapine, imipramine, and paroxetine. All of the trazodone patients were receiving it for sleep. The patient receiving imipramine was receiving it to treat urinary symptoms. The individual dosing are demonstrated in Table 3. In those patients receiving both antidepressant and procarbazine, the reason of mortality for discontinuation of procarbazine was higher than those patients receiving procarbazine alone, although this was not statistically significant.

\section{Evaluation of serotonin toxicity}

No patients were positive for ST according to Hunter's criteria. Diarrhea, tremor, and shivering were the symptoms from Sternbach's criteria that were further analyzed, because these symptoms are not likely to be related to the patient's condition but may be more closely associated with antidepressant use. No patients were positive for hyperreflexia or hypomania. Patients who were positive for agitation, diaphoresis, ataxia, confusion, or myoclonus had many other confounding factors and were deemed by the provider in the medical chart to be multifactorial and/ or due to disease, infection or other causes. Of the patients who received an antidepressant concomitantly with their procarbazine, diarrhea occurred $8.54 \%$ of the time, tremor occurred $5.53 \%$ of the time, and shivering occurred $2.51 \%$ of the time. Tremor and shivering occurred simultaneously $1.01 \%$ of the time, diarrhea and tremor occurred together $0.5 \%$ of the time, and all three occurred $0.5 \%$ of the time. Although the presence of all three occurred $0.5 \%$ of the time, other etiologies could not be ruled out. Thus, the diagnosis of serotonin toxicity according to Sternbach's criteria was determined to be unlikely. There were a total of 18 patients that experienced diarrhea, tremor, or shivering. Nine of these patients were on an antidepressant concomitantly with their procarbazine and 8 of the 18 patients had a brain tumor as their cancer diagnosis. Three of these nine patients were taking citalopram, two were taking trazodone, and the remaining patients were individually taking sertraline, paroxetine, venlafaxine and mirtazapine. Ten of 18 patients had stage 4 diagnosis, and 5 of the 18 had received four or more prior cancer treatments. The 
Table 2. Clinical characteristics of patients who received procarbazine and an antidepressant compared with those who received procarbazine monotherapy

\begin{tabular}{|c|c|c|c|}
\hline \multirow[b]{2}{*}{ Characteristic } & \multicolumn{3}{|c|}{$\begin{array}{l}\text { Procarbazine }+ \\
\text { antidepressant Procarbazine }\end{array}$} \\
\hline & $N=26$ & $N=39$ & $P$-value \\
\hline \multicolumn{4}{|l|}{ Gender (\%) } \\
\hline Male & 61.5 & 82.8 & 1.0000 \\
\hline Female & 38.5 & 17.2 & \\
\hline Mean age (years) & $54.9 \pm 18.44$ & $59.5 \pm 15.45$ & 0.2800 \\
\hline \multicolumn{4}{|l|}{ Diagnosis (\%) } \\
\hline Brain tumor & 38.5 & 51.3 & 0.4465 \\
\hline Lymphoma & 61.5 & 48.7 & \\
\hline \multicolumn{4}{|l|}{ Stage at diagnosis (\%) } \\
\hline Stage 1 & 0.0 & 2.6 & 1.0000 \\
\hline Stage 2 & 26.9 & 25.6 & 1.0000 \\
\hline Stage 3 & 19.2 & 23.1 & 0.7678 \\
\hline Stage 4 & 53.9 & 51.3 & 0.8014 \\
\hline \multicolumn{4}{|c|}{ Number of prior chemotherapy treatments (\%) } \\
\hline No prior treatment & 19.2 & 5.1 & 0.1059 \\
\hline I prior treatment & 15.4 & 28.2 & 0.3679 \\
\hline 2 prior treatments & 38.5 & 30.8 & 0.5970 \\
\hline 3 prior treatments & 7.7 & 12.8 & 0.6928 \\
\hline$\geq 4$ prior treatments & 19.2 & 23.1 & 0.7678 \\
\hline \multicolumn{4}{|l|}{ Type of antidepressant (\%) } \\
\hline SSRI & 53.8 & $\mathrm{n} / \mathrm{a}$ & \\
\hline SNRI & 19.2 & $\mathrm{n} / \mathrm{a}$ & \\
\hline TCA & 3.8 & $\mathrm{n} / \mathrm{a}$ & \\
\hline MAOI & 0.0 & $\mathrm{n} / \mathrm{a}$ & \\
\hline Other & 23.2 & $\mathrm{n} / \mathrm{a}$ & \\
\hline $\begin{array}{l}\text { Dose per day (mg) } \\
\text { of procarbazine (median) }\end{array}$ & $50.0(35.7-64.8)$ & $\begin{array}{c}50.0(37.5- \\
67.3)\end{array}$ & 0.5169 \\
\hline $\begin{array}{l}\text { Number of procarbazine } \\
\text { cycles (median) }\end{array}$ & $2.0(1.0-4.0)$ & $2.0(1.0-3.5)$ & 0.4868 \\
\hline \multicolumn{4}{|c|}{ Reason for discontinuation of procarbazine (\%) } \\
\hline Death & 26.9 & 7.7 & 0.0751 \\
\hline Progression of disease & 42.3 & 66.7 & 0.0741 \\
\hline Completion of treatment & 3.8 & 2.6 & 1.0000 \\
\hline Intolerability & 23.1 & 23.0 & 1.0000 \\
\hline Unknown & 3.9 & 0.0 & 0.4000 \\
\hline
\end{tabular}

SSRI, selective serotonin reuptake inhibitor; SNRI, serotonin and norepinephrine reuptake inhibitor; TCA, tricyclic antidepressant; MAOI, monoamine oxidase inhibitor.

average dose of procarbazine for these 18 patients was $73.1 \pm 71.2 \mathrm{mg} /$ day each cycle. Reason for discontinuation of the procarbazine was progression of disease (44.44\%), intolerability $(33.33 \%)$, death $(16.67 \%)$, or completed treatment $(5.56 \%)$. Of the patients who discontinued procarbazine due to intolerability, none were due to diarrhea, tremor, or shivering. There was no correlation between these symptoms and diagnosis, procarbazine dose, number of prior chemotherapy cycles, nor stage at diagnosis.

\section{Discussion}

No patients in the study were diagnosed with serotonin toxicity, nor did any meet Hunter's diagnostic criteria for serotonin toxicity. There were several limitations to this study. First, our sample size was small because procarbazine is not a first-line chemotherapy agent, and its use is relatively uncommon. Second, because this was a retrospective study, patient evaluation was limited to the notes recorded in medical records. A prospective design would allow a far more thorough assessment of signs and symptoms of serotonin toxicity. This would also allow for more accurate start and stop dates of procarbazine, antidepressants, and other medications. Another consideration is the significant overlap in symptoms of progression of central nervous system (CNS) cancers and serotonin toxicity. CNS malignancies often cause patients to exhibit confusion, agitation, seizures, tremor , and incoordination, all features consistent with ST. We collected stage at diagnosis to further delineate if symptoms were related to advanced disease versus a potential drug interaction. Distinguishing ST in patients on chemotherapy is even more difficult, as anticancer drugs can elicit additional ST-like symptoms such as nausea, diarrhea, and changes in blood pressure. Had there been severe symptoms concerning for an interaction, we expect that these would have been reported in the medical record. Because symptoms such as nausea and diarrhea can be dose related with procarbazine [19], our finding that there wasn't a statistically significant difference in mean dose between those with or without an antidepressant further supports that nausea and diarrhea were due to disease or other factors and less likely related to a drug interaction. Although mild symptoms of ST may have occurred without being attributed to the procarbazine-antidepressant drug interaction, the authors feel procarbazine can be safely used concomitantly with first-line antidepressants, provided patients are appropriately monitored.

In addition to the symptom overlap, a number of conditions exhibit features similar to ST, including neuroleptic malignant syndrome, anticholinergic delirium, CNS infection, and malignant hyperthermia. While evaluating charts for ST symptoms, these differential diagnoses were

Table 3. Average doses of antidepressants

\begin{tabular}{|c|c|c|c|c|c|c|}
\hline & Fluoxetine (I) & Citalopram (6) & Escitalopram (2) & Paroxetine (I) & Sertraline (3) & Trazodone (7) \\
\hline \multirow[t]{2}{*}{ Average daily dose } & 20 mg & $20 \pm 10 \mathrm{mg}$ & $7.5 \pm 2.5 \mathrm{mg}$ & $20 \mathrm{mg}$ & 50 mg & $50 \pm 25 \mathrm{mg}$ \\
\hline & Duloxetine (I) & Venlafaxine (4) & Mirtazepine (I) & Imipramine (I) & Amitriptyline (I) & \\
\hline Average daily dose & 60 mg & $168.75 \pm 94.37 \mathrm{mg}$ & $7.5 \mathrm{mg}$ & $25 \mathrm{mg}$ & $10 \mathrm{mg}$ & \\
\hline
\end{tabular}


considered and ruled out. The authors did not examine specific diagnosis of infection and steroid-induced psychosis. Because patients on immunosuppressive medications are at increased risk for infection and are frequently treated with steroids, these should have been considered during data collection. However, when symptoms of either condition appeared these were noted. Although not statistically significant, the mortality rate was higher in patients who were receiving both procarbazine and an antidepressant. It is difficult to explain this result due to the retrospective nature of the study and the small sample size with the procarbazine with an antidepressant group having the smaller denominator compared with the procarbazine alone group. Additionally, the procarbazine with an antidepressant group has more lymphoma patients than the procarbazine alone group. Procarbazine is often used in patients in lymphoma who cannot tolerate traditional therapy due to comorbidities and who have exhausted other treatment modalities [20,21].

One important observation made while performing this study was the counseling provided to patients starting procarbazine. Multiple chart notes indicated that patients were instructed to follow strict dietary limitations, including avoidance of cheese, chocolate, and alcohol. The intention is presumably to limit tyramine, an amine whose metabolism is inhibited by procarbazine and accumulation can lead to hypertensive crisis. The accuracy and consistency of education regarding tyramine restriction varied significantly among prescribers. Several factors may limit the risk of dietary tyramine to patients receiving procarbazine. One such factor includes the extent to which procarbazine inhibits monoamine oxidase, which is unclear but appears to be relatively low. Another aspect of procarbazine treatment that may reduce the risk of these foods is the cyclical schedule that is utilized in many procarbazine-containing chemotherapy regimens. Short cycles are likely to give the body time to eliminate excessive tyramine. Instructing patients to avoid foods that they enjoy can further reduce their quality of life. It is also necessary to consider the nutritional deficiencies of patients receiving chemotherapy or with cancers that reduce appetite. Patients often find eating difficult, and adding unnecessary restrictions or associating food with fear or danger may increase the risk for nutritional deficits. It is unclear if this education on tyramine restriction is necessary, and one paper suggests that dietary restrictions are not clinically indicated [22].

Another practice that was occasionally included in chart notes was instructing patients to hold antidepressants during procarbazine treatment. This suggestion stems from the need to stop all antidepressants at least 2 weeks prior to initiation of an MAOI, with the exception of fluoxetine, which requires at least a 5-week washout period due to the long half-life, to avoid potentially fatal consequences like serotonin syndrome or hypertensive crisis [12]. The documentation indicated that some patients were told to stop their antidepressant ' 15 days around' procarbazine, and others were advised to stop taking the antidepressant the day procarbazine was initiated without mention of a taper in order to discontinue the antidepressant. Many patients on antidepressants appear to have been given no instruction to discontinue their antidepressant and were allowed to continue their medication during procarbazine treatment. The inconsistencies among these recommendations, as well as the appropriateness of these recommendations, are concerning. For example, if an antidepressant would be held at initiation, the washout would not be adequate, and it would likely not impact the risk of serotonin toxicity, if a true risk exists. Further, these examples highlight the needed for provider of education regarding the risk for the interaction as well as strategies to minimize this risk. Further, it should be noted that patients receiving procarbazine with or without an antidepressant should also be monitored for worsening or new psychiatric symptoms. The product labeling notes potential psychiatric effects of procarbazine include hallucinations, depression, nervousness, apprehension, nightmares, and confusion [23]. It is unclear if procarbazine itself may precipitate the need for an intervention to manage depression. Therefore, close monitoring is critical if procarbazine is initiated alone or in combination with an antidepressant, or if the antidepressant is discontinued in the setting of procarbazine use. The results of this study, although of a small sample, suggest that the risk of serotonin toxicity posed by the interaction between procarbazine and antidepressants is not likely worth the risk to a patient's mental health posed by discontinuing antidepressant treatment. With that said, if continuation of the patient's antidepressant treatment is not warranted at the time procarbazine is to be initiated, it would be judicious to abide by the recommended antidepressant washout periods.

\section{Conclusion}

In this small sample of patients treated with procarbazine plus an antidepressant (most typically SSRIs), there were no reports of serotonin toxicity observed, nor did any patient meet diagnostic criteria for ST. The authors urge clinicians to ensure depression is adequately managed in cancer patients who are undergoing procarbazine therapy, starting with typical first-line antidepressant agents or psychotherapy, as appropriate. Although there is a risk of serotonin toxicity due to procarbazine's weak MAOI activity, to our knowledge, there has been no clinical cases published reporting on such an interaction. Clinicians must assess the risk for drug interactions when making treatment decisions, as always. However, procarbazine's inhibition of monoamine oxidase should not dissuade prescribers from addressing a patient's depressive symptoms, whether it is with pharmacologic or behavioral therapy, ensuring close 
monitoring ensues and high risk antidepressants, like other monamine oxidase inhibitors, are avoided. Further, patients and caregivers should be provided with both verbal and written information detailing the signs and symptoms of serotonin toxicity, although the suspected risk is low.

\section{Conflict of interest}

The authors report no conflicts of interest. The authors alone are responsible for the content and writing of the paper.

\section{References}

1. Chu E, Sartorelli AC. Chapter 54. Cancer chemotherapy. In Basic \& Clinical Pharmacology (12th edn), Katzung BG, Masters SB, Trevor AJ (eds), McGraw-Hill: New York, 2012. http://www.accesspharmacy.com/content.aspx? $\mathrm{aID}=55831105$. [Accessed July 10, 2013].

2. Holt A, Callingham BA. Further studies on the ex-vivo effects of procarbazine and monomethylhydrazine on rat semicarbazidesensitive amine oxidase and monoamine oxidase activities. $J$ Pharm Pharmacol 1995; 47:837-845.

3. Micromedex ${ }^{\circledR}$ Healthcare Series [Internet database]. Greenwood Village, Colo: Thomson Healthcare. Updated periodically. Available at: http://www.micromedexsolutions.com/home/ dispatch [Accessed December 19, 2012].

4. Lexi-Comp Online, [Internet database] Hudson, Ohio: Lexi-Comp, Inc. Available at: http://www.crlonline.com/lco/action/home/ switch [Accessed December 19, 2012].

5. Facts \& Comparisons. [Internet database] St. Louis, MO: Wolters Kluwer Health, Inc. Available at: http://online.factsandcomparisons.com [Accessed December 19, 2012].

6. Epocrates online. [Internet database] San Mateo, CA: Epocrates, Inc. Available at: https://online.epocrates.com/ [Accessed December 19, 2012].

7. Roper K, McDermott K, Cooley M, Daley K, Fawcett J. Health-related quality of life in adults with Hodgkin's disease: the state of the science. Cancer Nurs 2009;32:E1-E17.

8. Kessler RC, Chiu WT, Demler O, Walters EE. Prevalence, severity, and comorbidity of twelve-month DSM-IV disorders in the National Comorbidity Survey Replication (NCS-R). Arch Gen Psychiatry 2005;62:617-627.

9. Wang PS, Lane M, Olfson M, Pincus HA, Wells KB, Kessler RC. Twelve month use of mental health services in the United States. Arch Gen Psychiatry 2005;62:629-640.

10. Reich M. Depression and cancer: recent data on clinical issues, research challenges and treatment approaches. Curr Opin Oncol 2008;20:353-359.

11. Davidson JRT. Major depressive disorder treatment guidelines in America and Europe. $J$ Clin Psychiatry 2010;71(Suppl E1):e04.

12. American Psychiatric Association. Practice guideline for the treatment of patients with major depressive disorder, 3rd edition. 2010. Available at: http://psychiatryonline.org/content. aspx ?bookid $=28 \&$ sectionid $=1667485$ [Accessed December 19, 2012].

13. Mason PJ, Morris VA, Balcezak TJ. Serotonin syndrome: presentation of 2 cases and review of the literature. Medicine 2000;79:201-209.

14. Bronstein AC, Spyker DA, Cantilena LR, Rumack BH, Dart RC. 2011 Annual report of the American Association of Poison Control Centers' national poison data system (NPDS): 29th annual report. Clin Toxicol (Phila) 2012;50:911-1164.
15. Sternbach $\mathrm{H}$. The serotonin syndrome. Am $J$ Psychiatry 1991;148(6):705-713.

16. Boyer EW. Serotonin syndrome. In UpToDate, Traub SJ, Grayzel J (eds), UpToDate: Waltham, MA, 2013.

17. Dunkley EJ, Isbister GK, Sibbritt D, Dawson AH, Whyte IM. The hunter serotonin toxicity criteria: simple and accurate diagnostic decision rules for serotonin toxicity. QJM 2003;96:635-642.

18. Isbister G, Buckley N, Whyte I. Serotonin toxicity: a practical approach to diagnosis and treatment. Med J Aust 2007;187:361-365.

19. van Eys J, Cangir A, Pack R, Baram T. Phase I trial of procarbazine as a 5-day continuous infusion in children with central nervous system tumors. Cancer Treat Rep 1987;71:973-974.

20. National Comprehensive Cancer Network. Central Nervous System Cancers (Version 2.2013). http://www.ncen.org/professionals/ physician_gls/pdf/cns.pdf [Accessed July 10, 2013].

21. National Comprehensive Cancer Network. NonHodgkin's Lymphoma (Include Version 1.2013). http://www.nccn.org/professionals/ physician_gls/pdf/nhl.pdf [Accessed July 10, 2013].

22. Maxwell MB. Reexamining the dietary restrictions with procarbazine (an MAOI). Cancer Nurs 1980;3:451-457.

23. Matulane (B) [package insert]. Gaithersburg, MD; Sigma-tau Pharmaceuticals, Inc.; 2008. 